\title{
Low-loss, low-cross-talk crossings for silicon-on-insulator nanophotonic waveguides
}

\author{
Wim Bogaerts, * Pieter Dumon, Dries Van Thourhout, and Roel Baets \\ Ghent University - IMEC, Department of Information Technology, Photonics Research Group, \\ Sint-Pietersnieuwstraat 41, 9000 Gent, Belgium \\ *Corresponding author: wim.bogaerts@intec.ugent.be
}

Received July 25, 2007; accepted August 14, 2007;

posted August 30, 2007 (Doc. ID 85702); published September 20, 2007

We present compact crossings for silicon-on-insulator photonic wires. The waveguides are broadened using a $3 \mu \mathrm{m}$ parabolic taper in each arm. By locally applying a lower index contrast using a double-etch technique, loss of confinement is reduced and $97.5 \%$ transmission $(-1.7 \mathrm{~dB})$ is achieved with only $-40 \mathrm{~dB}$ cross talk. (C) 2007 Optical Society of America

OCIS codes: $130.0130,130.1750,130.2790$.

With silicon-on-insulator (SOI) photonic wires one can combine many compact functions into a photonic integrated circuit $[1,2]$. The high refractive index contrast (3.45 to 1.45) enables tightly confined waveguides with a submicrometer core. In addition, one can use commercial silicon electronics fabrication technology for low-cost, large-scale manufacturing. Current propagation losses of about $2.5 \mathrm{~dB} / \mathrm{cm}$ [1] allow waveguides of several centimeters in length, so already situations arise where the integration of more functions is being limited by the topology of the interconnections. Contrary to electronics, where many-layered interconnects allow flexible routing, current SOI photonic circuits are constricted to a single layer. Without low-loss, low-cross-talk waveguide crossings, routing of more complex photonic circuits is difficult or even impossible.

When using a direct waveguide crossing, lateral confinement in the photonic waveguide is lost near the crossing, causing diffraction of the light. In lowcontrast waveguides these direct waveguide crossings are just a minor perturbation of the straight waveguide, even though the the crossing is several micrometers long. In this situation, the beam is hardly diffracted. This does not hold in high-contrast systems such as SOI, where the guided mode in a photonic wires has wide-angle spatial components. At a direct crossing, the mode diffracts dramatically and a sizable fraction of the light is radiated away. Calculations and experiments show losses between -1.1 and $-1.4 \mathrm{~dB}$ (72\% transmission) per crossing [3,4], and this depends strongly on the wire width. Also, this generates significant cross talk of approximately $-9 \mathrm{~dB}$ in the arms of the crossing. We improve the transmission of the crossing to $-0.16 \mathrm{~dB}$ by expanding the waveguide mode, while at the same time locally reducing the refractive index contrast. This also reduces the cross talk to a level of $-40 \mathrm{~dB}$.

To improve the transmission to the other waveguide one can either aim to reduce the diffraction in the crossing region $[4,5]$ or use a resonant cavity that couples to the correct waveguide [6]. By using a resonance, the second technique will impose a strong wavelength dependence on the crossing. Therefore, we try to suppress the diffraction by suppressing the wide-angle components of the mode. Ideally, the mode is transformed into a something resembling a plane wave (or slab mode) near the crossing. For this, we broaden the waveguide core, which can be done using elliptical or parabolic mode expanders as used by Fukazawa et al. [4] and shown in Figs. 1(b) and 1(c). However, in the case of a fourfold symmetric crossing [Fig. 1(c)], the widening of the core also increases the length of the unconfined region, causing even higher losses. Indeed, in [4] a fourfold symmet-
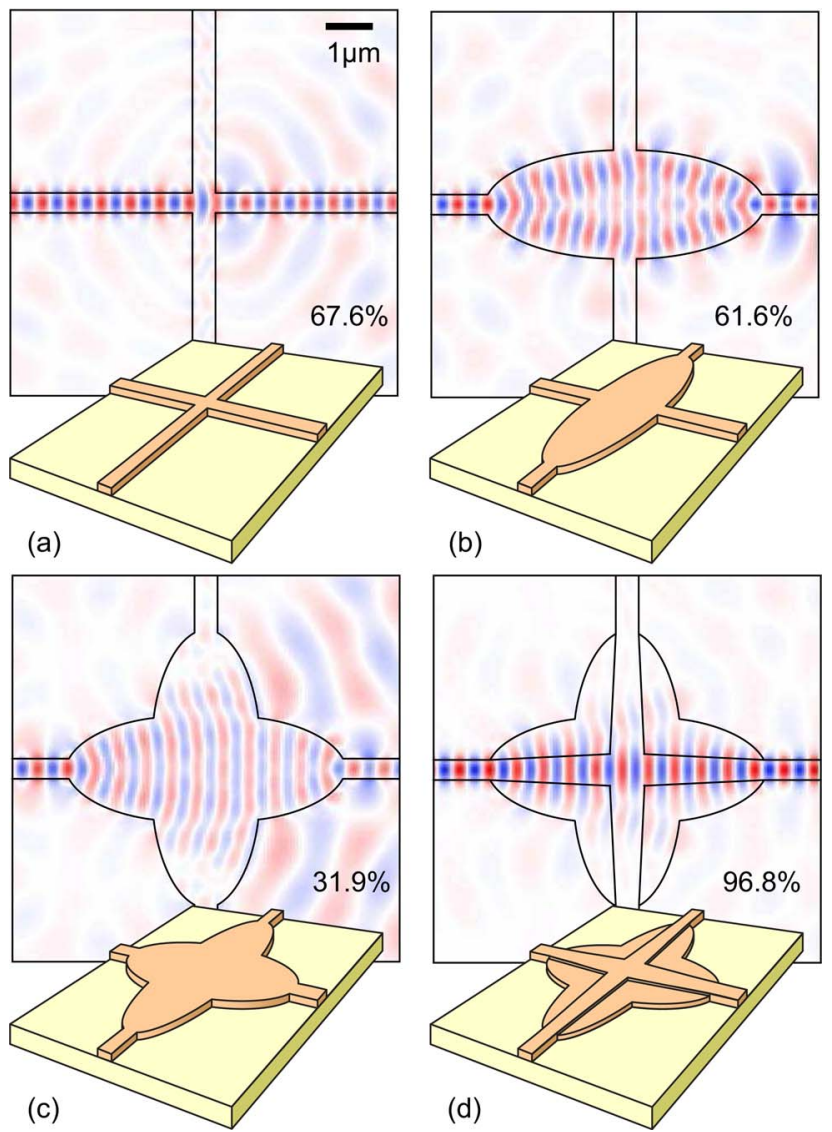

Fig. 1. (Color online) 2-D simulations of waveguide crossings: (a) direct crossing, (b) twofold symmetric elliptical crossing [4] with a $6 \mu \mathrm{m}$ length, (c) elliptical crossing [4] with a $6 \mu \mathrm{m}$ length, (d) crossing from Fig. 1. 
ric crossing is calculated to have a $-0.4 \mathrm{~dB}$ loss, while a crossing with only one expanded waveguide has only $-0.1 \mathrm{~dB}$ loss. By tailoring the mode distribution in the expansion area, e.g., by using a multimode interferometer, as in [3], the diffraction losses can be reduced, because more wide-angle components can be removed. Still, such designs require crossing lengths of at least $10 \mu \mathrm{m}$.

To reduce the diffraction losses in the crossing region, we introduce a scheme to maintain confinement over a larger part of the expanded crossing, which at the same time lowers the lateral refractive index contrast. The lower index contrast reduces the diffraction in the nonconfined region, as well as backreflections. The lower lateral index contrast of the waveguide is achieved through a double-etch scheme [1], shown in Fig. 2. The high-contrast photonic wires and the parabolic mode expanders are etched through the silicon core, but a shallow-etched crossing confines the light to the center of the mode expander. This technique has several positive effects on the crossing. First, confinement is maintained over a larger part of the crossing. Second, the lower index in the shallow-etched regions has the effect that the curved phase fronts in the expansion region are straightened. This suppresses wide-angle components in the mode distribution. Third, by using a

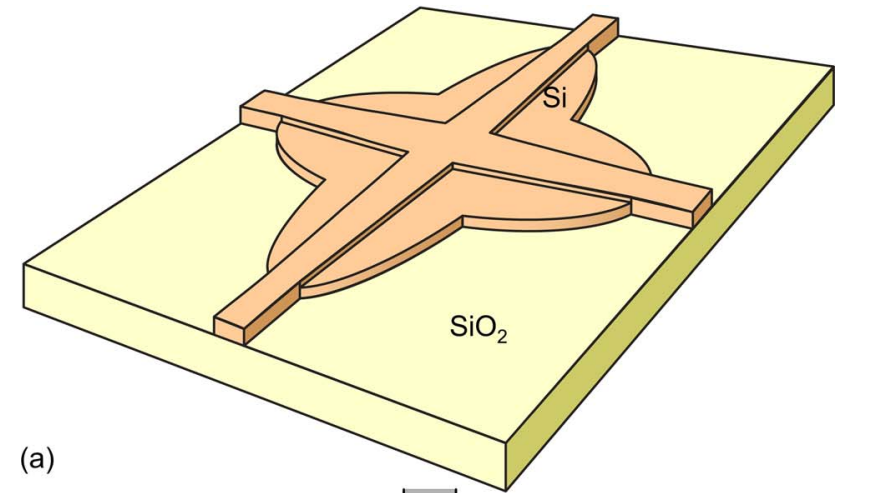

(a)

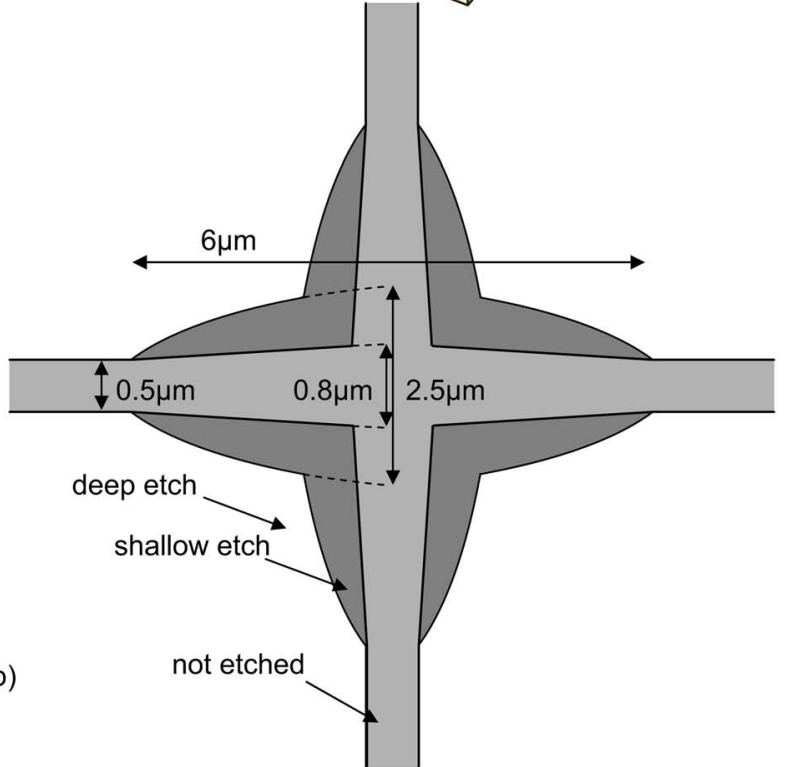

Fig. 2. (Color online) Waveguide crossing with a double etch. lower-index contrast, backreflections in the crossing are reduced.

The simulation results from Fig. 1 illustrate this. 2-D eigenmode expansion was used at a wavelength of $1.55 \mu \mathrm{m}$. The direct crossing [Fig. 1(a)] has only $67 \%$ transmission and several percent of cross talk in the arms. This is in good agreement with [4]. The twofold symmetric elliptical crossing [Fig. 1(b)] was designed according to [4], but with only $6 \mu \mathrm{m}$ length, which reduces the transmission to $61 \%$. This is even more dramatic for the fourfold symmetric elliptical crossing [Fig. 1(c)], which has only $32 \%$ transmission, because of the larger loss of confinement. The doubleetch scheme [Fig. 1(d)] can compensate for this, which results in a transmission of over $95 \%$.

We fabricated the circuits on commercial $200 \mathrm{~mm}$ SOI wafers with $220 \mathrm{~nm}$ of top silicon and $2 \mu \mathrm{m}$ of oxide. For the pattern definition we used $248 \mathrm{~nm}$ deep UV lithography and dry etching [1]. The shallowetched structures are defined first, followed by the deeply etched waveguides. Alignment between the two process layers is done by the lithography tool with an accuracy of better than $50 \mathrm{~nm}$. The fabricated device is shown in Fig. 3.

As shown in Fig. 1, the crossing has an overall length of $6 \mu \mathrm{m}$, and the parabolic expander would reach a center width of $2.5 \mu \mathrm{m}$. The shallow-etched waveguide expands linearly from 500 to $800 \mathrm{~nm}$ over the length of the crossing. For characterization, we used vertical grating fiber couplers [1,7], which allow for easy alignment reproducible coupling efficiencies. We measured waveguides with $0,5,10$, and 21 crossings. The transmission characteristics as a function of wavelength are shown in Fig. 4(a). The Gaussian shape of the curves is characteristic for the grating fiber coupler gratings and is not a feature of the
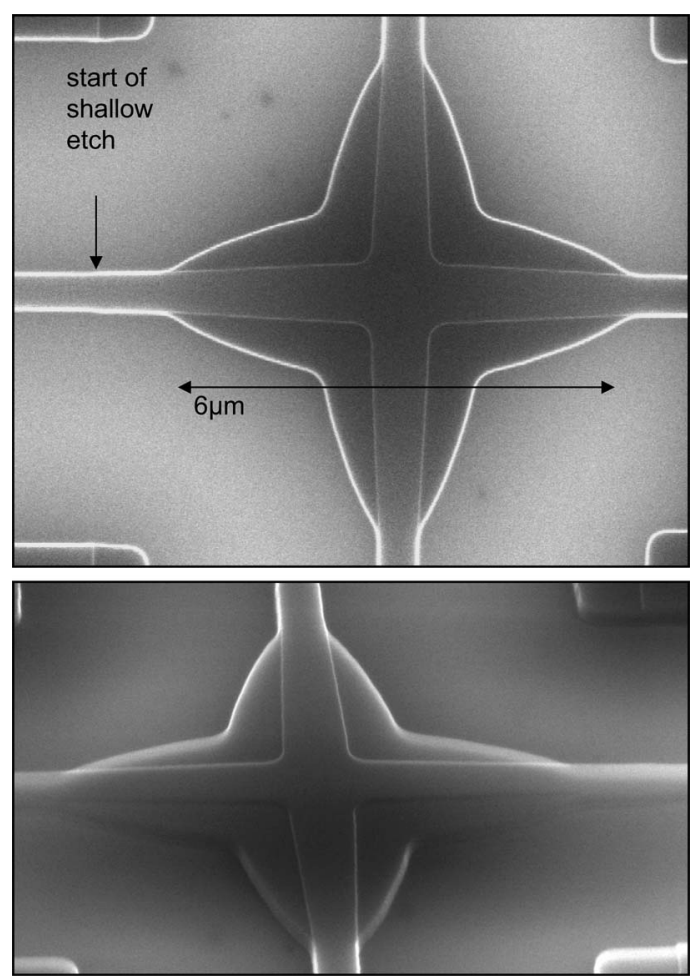

Fig. 3. Fabricated waveguide crossing. 

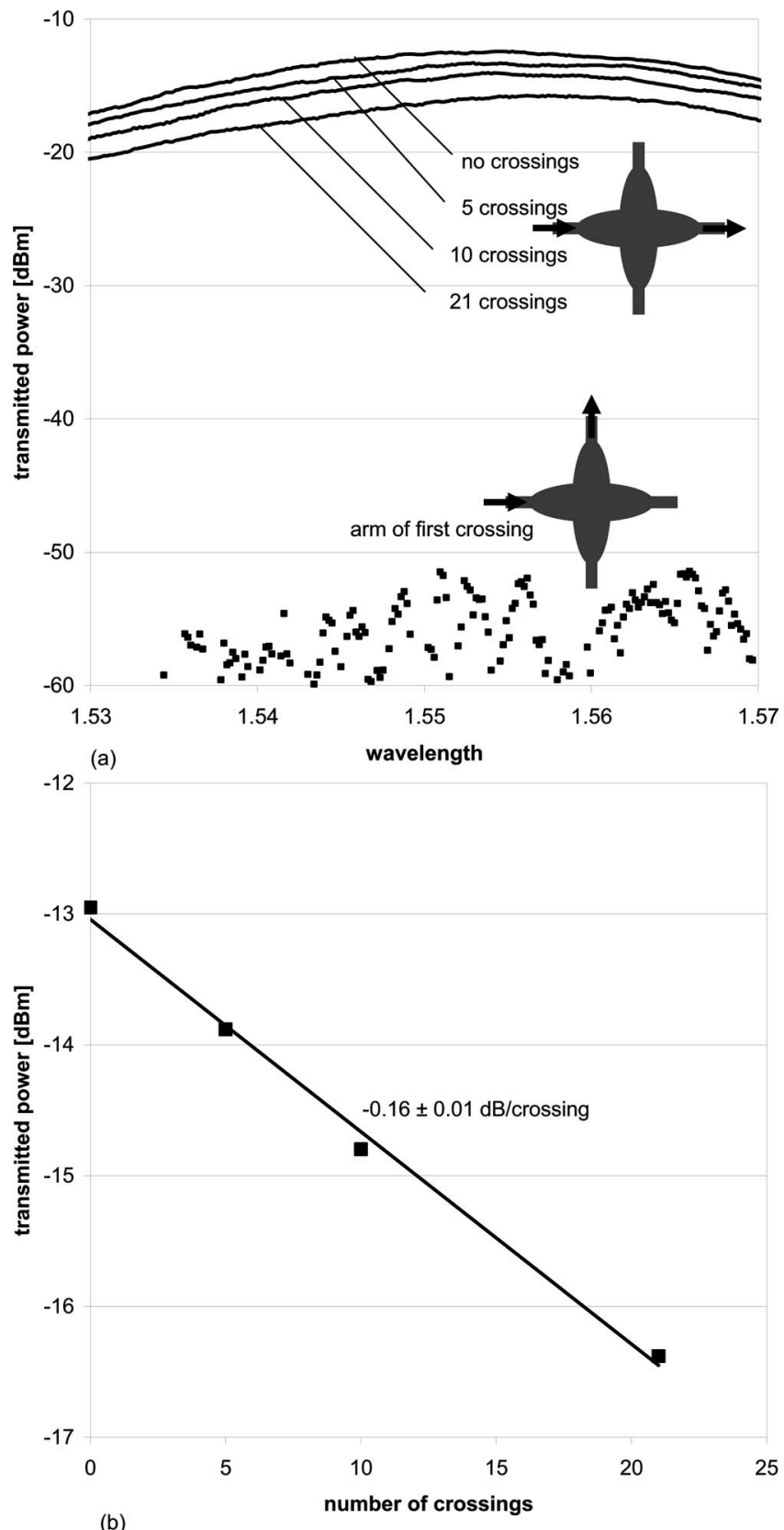

Fig. 4. Transmission measurements on multiple waveguide crossings: (a) transmission for $0,5,10$, and 21 crossings. The parabolic spectrum is typical for grating fiber couplers [7]. (b) Fitted loss per crossing at $1550 \mathrm{~nm}$. crossings [7]. In Fig. 4(b) we extract the transmission per crossing using a fit on the measurements at a wavelength of $1550 \mathrm{~nm}$. The loss per crossing is $-0.16 \mathrm{~dB}$, which amounts to $97.5 \%$ transmission. We also extracted an upper value for the cross talk by measuring the output from the side arm of the first crossing. We found the cross talk to be better than $-40 \mathrm{~dB}$. We also repeated the measurements for different waveguide widths between 440 and $550 \mathrm{~nm}$ and found the performance to be the similar, making the crossings quite tolerant to fabrication variations.

We have shown low-loss, low-cross-talk crossings for SOI photonic wire waveguides. The design uses parabolically broadened waveguides and a doubleetch scheme to reduce lateral refractive index contrast while still confining the light as much as possible in the crossing region. We measured $-0.16 \mathrm{~dB}$ loss and $-40 \mathrm{~dB}$ cross talk.

W. Bogaerts acknowledges a postdoctoral fellowship from the Flemish Research Foundation. This work was partly supported by the European Union through the IST-ePIXnet Network of Excellence and the Silicon Photonics Platform and partly supported by the Belgian Interuniversity Attraction Pole (IAP)PHOTON and the IAP-Photonics@be project.

\section{References}

1. W. Bogaerts, R. Baets, P. Dumon, V. Wiaux, S. Beckx, D. Taillaert, B. Luyssaert, J. Van Campenhout, P. Bienstman, and D. Van Thourhout, J. Lightwave Technol. 23, 401 (2005).

2. W. Bogaerts, P. Dumon, D. Van Thourhout, D. Taillaert, P. Jaenen, J. Wouters, B. S. V. Wiaux, and R. Baets, IEEE J. Sel. Top. Quantum Electron. 12, 1394 (2006).

3. H. Chen and A. Poon, IEEE Photon. Technol. Lett. 18, 2260 (2006).

4. T. Fukazawa, T. Hirano, F. Ohno, and T. Baba, Jpn. J. Appl. Phys., Part 1 43, 646 (2004).

5. C. Wei, F. Groen, M. Smit, I. Moerman, P. Van Daele, and R. Baets, J. Lightwave Technol. 15, 906 (1997).

6. S. Johnson, C. Manolatou, S. Fan, P. Villeneuve, J. Joannopoulos, and H. Haus, Opt. Lett. 23, 1855 (1998).

7. D. Taillaert, W. Bogaerts, P. Bienstman, T. Krauss, P. Van Daele, I. Moerman, S. Verstuyft, K. De Mesel, and R. Baets, IEEE J. Quantum Electron. 38, 949 (2002). 